\title{
$\Delta$ 9-Tetrahydrocannabinol Toxicity and Validation of Cannabidiol on Brain Dopamine Levels: An Assessment on Cannabis Duplicity
}

\author{
Swapnali Chetia ${ }^{1}\left[\right.$ Gaurab Borah $^{1}$
}

Received: 22 July 2020 / Accepted: 23 August 2020 / Published online: 28 August 2020

(c) The Author(s) 2020

\begin{abstract}
$\Delta$ 9-tetrahydrocannabinol (THC) of cannabis is the main psychoactive component which is a global significant concern to human health. Evaluation on THC reported its drastic effect on the brain dopaminergic (DAergic) system stimulating mesolimbic DA containing neurons thereby increasing the level of striatal DA. Cannabidiol (CBD), with its anxiolytic and anti-psychotic property, is potent to ameliorate the THC-induced DAergic variations. Legal authorization of cannabis use and its analogs in most countries led to a drastic dispute in the elicitation of cannabis products. With a recent increase in cannabis-induced disorder rates, the present review highlighted the detrimental effects of THC and the effects of CBD on THC induced alterations in DA synthesis and release. Alongside the reported data, uses of cannabis as a therapeutic medium in a number of health complications are also being briefly reviewed. These evaluated reports led to an anticipation of additional research contradictory to the findings of THC and CBD activity in the brain DAergic system and their medical implementations as therapeutics.
\end{abstract}

Swapnali Chetia

swapnali.chetia@rgu.ac.in; swapnalimann@gmail.com

1 Department of Zoology, Rajiv Gandhi University, Rono Hills, Doimukh, Arunachal Pradesh 791112, India 


\section{Graphic Abstract}

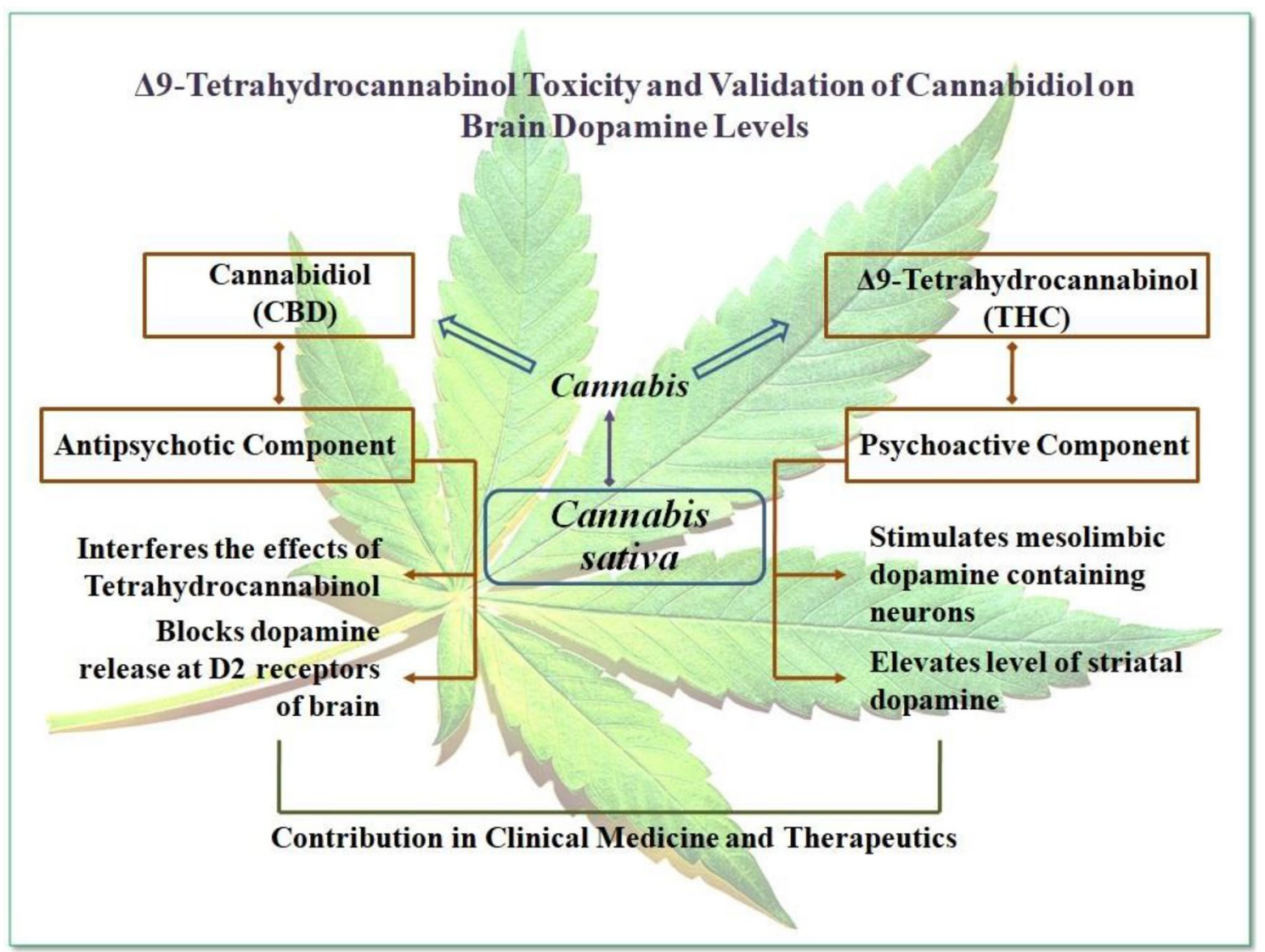

Keywords Cannabis · Tetrahydrocannabinol · Cannabidiol $\cdot$ Dopamine $\cdot$ Medicinal uses

$\begin{array}{ll}\text { Abbreviations } & \\ \text { AMPH } & \text { Amphetamine } \\ \text { AST } & \text { Associative striatum } \\ \text { CBD } & \text { Cannabidiol } \\ \text { CB1R and CB2R } & \text { Endocannabinoid Receptor 1 and 2 } \\ \text { DA } & \text { Dopamine } \\ \text { DOPAC } & \text { 3,4-Dihydroxy-phenylacetic acid } \\ \text { GABA } & \text { Gamma-aminobutyric acid } \\ \text { LST } & \text { Limbic striatum } \\ \text { mTOR } & \text { Mammalian target of rapamycin } \\ \text { MRS } & \text { Magnetic resonance spectroscopy } \\ \text { NAc } & \text { Nucleus accumbens } \\ \text { PET } & \text { Positron emission tomography } \\ \text { PFC } & \text { Prefrontal cortex } \\ \text { SMST } & \text { Sensorimotor striatum } \\ \text { THC } & \text { Tetrahydrocannabinol } \\ \text { VTA } & \text { Ventral tegmental area }\end{array}$

\section{Introduction}

Cannabis, commonly called marijuana, is a psychoactive and one of the most illicit recreational drugs around the world $[1,2]$, extracted from Cannabis sativa. With a precise estimation, as reported in 2017 , there are about 188 million cannabis users in the world, rating the world's population at approximately $3.8 \%$ [3]. Cannabis possesses antioxidant properties which may also contribute to other therapeutic aspects like anticonvulsant, anti-inflammatory, and neuroprotection, other than its detrimental effects [4]. Acute and chronic use of cannabis can influence CNS and PNS through several complications which include hyperemesis syndrome, impaired coordination, and performance, anxiety, psychotic disorders, suicidal tendencies, cannabis withdrawal symptoms, neurocognitive impairment, cardiovascular, respiratory, Cerebro-peripheral vascular diseases [1, 5], bullous lung disease, pneumothorax, desquamated interstitial disease, pneumopericardium, pneumomediastinum, and brown pigmented macrophages [6]. 
Apart from the virtue of medicinal properties, recent studies on chronic cannabis inhalation reported the intimation of cerebrovascular diseases [7] although the underlying mechanisms have not been strongly established yet. Numerous neurological disorders have been observed in discrete studies on cannabis exposure viz. cognitive dysfunction, behavioral complications, memory/attention deficiency, structural and functional variations in the brain [8-11]. About 100 cannabinoids are being reported to date [12], out of which $\Delta 9$ - tetrahydrocannabinol (THC) (Fig. 1) and cannabidiol (CBD) (Fig. 2) are the principal components that are actively involved in brain DAergic alterations. THC is one of the major and principal psychoactive constituents of cannabis [13]. In contrast, CBD is an anxiolytic and antipsychotic cannabinoid compound that may help in inhibiting the effects of THC and other negative effects effectuated due to cannabis exposure [14-16]. The psychoactive properties of THC are responsible for cannabis addictive potential and alterations in brain dopaminergic (DAergic) functions. Acute THC administration has been reported to elicit striatal dopamine (DA) release in animals [17] and humans [18-20].

\subsection{Cannabis Addiction}

Drug or cannabis addiction is a compulsive drug intake disorder, embodied due to loss of control over drug intake limitation and when prevented, emerges a negative emotional state [21]. THC is the principal causative cannabinoid responsible for cannabis addiction due to its psychoactive properties and associated effects on brain DAergic function. Koob and Volkow [21] described three stages of drug addiction that confer major alterations in neurocircuits. The three stages are-(a) the binge/intoxication stage generated by changes in basal ganglia characterized by excess impulsivity and irresistibility to drug usage despite the detrimental effects associated with it, (b) the withdrawal/negative affect stage driven by changes in the extended amygdala involving stria terminalis that implies reward cognition due to the loss of motivations towards non-drug rewards, (c) the

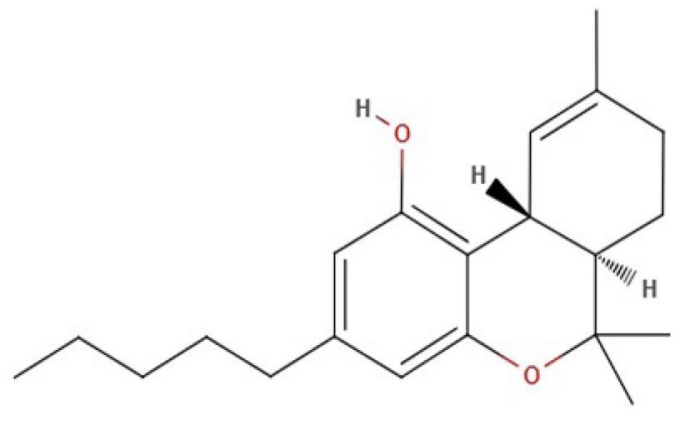

(a)

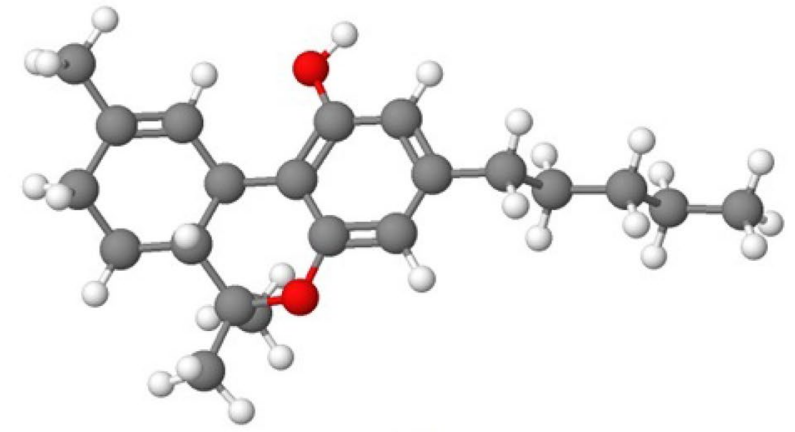

(b)

Fig. 1 Structural representation of $\Delta$ 9-tetrahydrocannabinol a structural arrangement $\mathbf{b} 3 \mathrm{D}$ - model of the structure

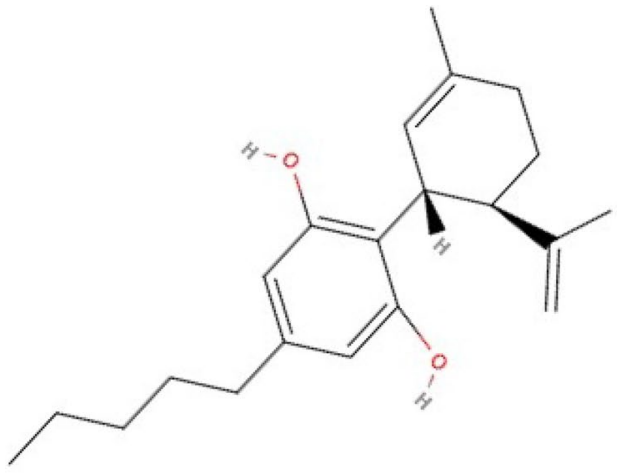

(a)

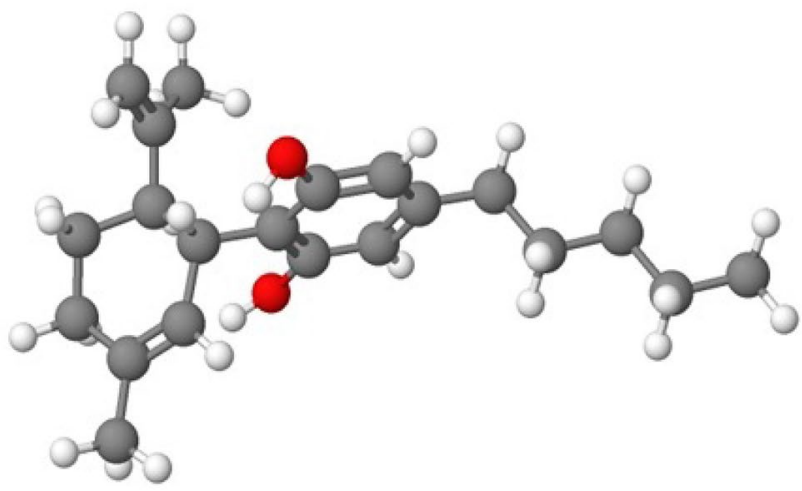

(b)

Fig. 2 Structural representation of cannabidiol a structural arrangement $\mathbf{b}$ 3D- model of the structure 
preoccupation/anticipation stage driven by alterations in the prefrontal cortex (PFC) involving disrupted GABAergic and glutamatergic activity (Fig. 3).

In the intoxication stage, the mesocorticolimbic DAergic reward pathway of the brain is hyperactivated followed by positive reinforcement of the rewarding effects of drugs. Impairment in incentive salience is a characteristic feature of the intoxication stage in which initial exposure to a drug intimating signaling contexts are exaggeratedly assigned with high rewarding properties. This lead to a conditioned evoke of DA release. Incentive salience dysfunction drives DA signals to perpetuate impulse of the drug upon exposure to conditioned-cues.

Following the intoxication stage, the withdrawal stage is triggered by adversary process responses following an overdosing interlude. These adversary process retaliations are marked by neurobiological within and between-system changes that direct motivation loss towards non-drug rewards and impaired emotion regulation. Within-system neuroconversions encompass dorsal striatum and the nucleus accumbens (NAc) with reduced DAergic signaling, resulting in elevation of reward thresholds for non-drug promoters, promoting depression. Between-system neuroadaptations embrace neurochemical dysfunction induced stress responses such as elevated extrication of corticotrophin-releasing factor (CRF) in the amygdala inclusive of HPA-axis dysfunction. These reverberates distinguishing symptoms specifically chronic irritability, anxiety-like responses, malaise, and dysphoria.

The preoccupation stage is involved in rehabilitationinduced abusive substance ensuing abstention. In this prolonged abstinence, effectuated constrain over craving and impulsivity is a vital strategy interposed by the PFC. This stage is marked by self-regulation, decision making, inhibitory control, and functional memory influenced through aberrant signaling between PFC and brain. This in turn might suspend the activity of GABAergic and glutamatergic exploitation. Proprietarily, this renders surplus salience incrimination of drug-induced cues, reduction in responsiveness-induced non-drug signaling intensifiers, and reduces maladaptive behavior inhibition activity.

\section{Cannabis and Dopamine}

Vulnerability to DA-enhancing substances of abuse is analogous to impaired striatal DA transmission [22, 23]. Studies in chronic cannabis users (CD) with co-morbid psychotic symptoms unveiled a reduction in DA synthesis [24] and mitigate the release of stress-induced DA [25]. This exhibits an inconsistent effect of reduced DA release in chronic cannabis users without psychotic symptoms. These inconsistent effects are relevant to concomitant use of cannabis along with other non-specified drugs, which depends on the

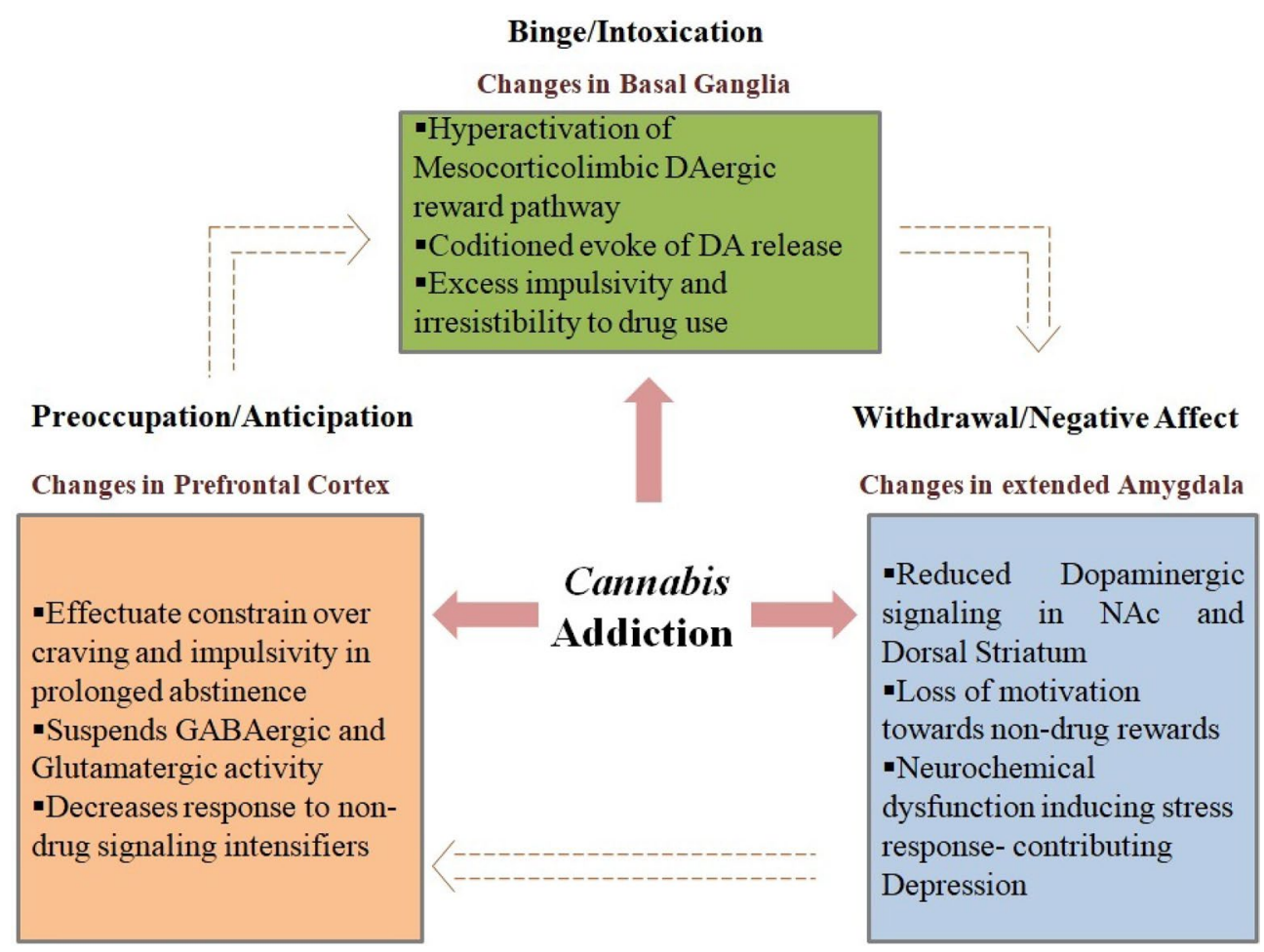

Fig. 3 Stages of drug addiction: binge/intoxication stage, withdrawal/negative affect stage and preoccupation/anticipation stage 
cannabis severity. Through positron emission tomography (PET) $[11 \mathrm{C}]$ raclopride, it is found that severity of cannabis use vary across cohort limitations of DA quantification changes. It was earlier demonstrated that the use of cannabis induces a deficit in neurocognitive function [26] and decreases striatal DA action [27, 28]. It was also predicted that in chronic cannabis users, reduction in DA release would counter-correlate with neurocognitive function and effectively assign psychosis-related symptoms [29].

It has been reported that upregulation of glutamate levels in the striatum and hippocampus could lead to schizophrenia $[30,31]$ and the use of cannabis induces psychosis [32]. In a cohort, evaluation of striatal-hippocampal glutamate levels and striatal DA release were juxtaposed through magnetic resonance spectroscopy (MRS) [29]. They highlighted that cannabis could alter glutamate levels in the hippocampus and striatum along with alterations in DA release. Thereby, reduced striatal DA release could promote psychopathology in cannabis users.

Although, it was demonstrated that, in cannabis users, reduced striatal DA release affects associative striatum (AST) and sensorimotor striatum (SMST) regions in contrast to other drugs that induce limbic striatum (LST) DA release deficit and effectuate intense seeking/craving [22, 23]. A possible reason could be the distribution of distinctive anatomical targets of THC, CB1 receptor. As reported, CB1 receptors are rarely present in the ventral striatum in contrast to the putamen or dorsal striatum in humans [33] or rat brain respectively $[34,35]$. Besides, it was also documented that, in humans, numerous CB1 receptors are present in the pallidus as compared to thalamus [36]. Reduction in striatal DA release, however, is relatable to a tedious performance in working memory [26]. Conversely, higher striatal DA release engenders intense performance of the same [27, 28]. It is eminently suggested that cannabis use in adolescence may promote drug dependence associated with a compromised DAergic system that may alter brain function.

\section{1 $\Delta$ 9-Tetrahydrocannabinol (THC) and Dopamine}

$\Delta 9$-tetrahydrocannabinol (THC) of cannabis is the principal psychoactive constituent that expends its effect on the brain. THC is an endocannabinoid receptor (CB1R) and CB2 receptor (CB2R) partial agonist, which is extensively embodied in the basal ganglia and SN pars reticulata of the brain $[36,37]$. THC stimulates mesolimbic DA containing neurons and elevates the level of striatal DA in animals [38]. In some studies, in humans, acute THC is reported to instigate striatal DA release $[19,39,40]$, but reports also exist that don't support this statement [41, 42].

CB1R antagonist rimonabant, through the activation of G-protein-coupled CB1R receptors, blocks the execution of psychoactive effects of THC [43, 44]. This mitigates the levels of cAMP by retarding the expression of adenylate cyclase [45]. THC disrupts the calibration of CB retrograde signaling systems on account of both its temporal and neuronal specificity over THC. Under conditions of a low density of CB1R, THC aggravates endogenous agonists procuring greater receptor efficacy than THC [46]. Through the allosteric activity, THC modulates opioid receptors [47], which may additionally dispense indirect routes for variations in DA transmission [48].

\subsubsection{CB1 Receptors and Dopamine}

Earlier, studies in animal models, demonstrated that amphetamine (AMPH) increases DA release when interacted with THC [49]. These reported that depending on the dose of THC, the behavioral effects of amphetamine may vary [50]. Indeed, contributing to the recreational and detrimental effects of cannabis, involuted THC generates complications on the DA system.

It is reported that the endocannabinoid complex modulates the functioning of the DAergic neuronal system [51]. Anandamide and 2-arachidonoylglycerol (2-AG) (endocannabinoid ligands) along with CB1Rs are abundantly present in the DAergic pathways [52]. Inevitably, they voluntarily exploit as a retrograde feedback system to regulate DA transmission on presynaptic GABAergic nerve terminals. These endocannabinoid ligands, consequently, vitalize the release of DA in the NAc shell [53]. CB1 antagonist rimonabant, stipulating endocannabinoids induced DAergic effects involving CB1R, blocks the modulation of this process. THC reward over increased DA release and DAergic neuronal discharge are reinforced by partial signal transduction mechanisms from CB1R [43].

\subsubsection{Studies in Acute THC and Dopamine}

In vitro radiolabelled studies of DA in synaptosomes of rodents revealed that THC induces increased DA synthesis [54] and release [50]. However, the effects of THC on DA activity laid a conflict with the evidence of both its elevation [55] and dose-dependent reduction [50]. Subsequently, it was reported that a lower dose of THC increases throughout the process of conversion from tyrosine to DA, whereas high doses resulted in decreased DA synthesis [56]. However, monitoring the correlation of THC administration and levels of DA alterations executed that repeated dosing of cannabis may result in behavioral and neurochemical fluctuations which may relevantly provoke drug dependence. In rodents, due to reduced precursor uptake [55] and $\mu$-opioid receptors-mediated DA-opioid reciprocity, reverberation of dose-specific THC is assumed to occur [54].

In an in vivo study, THC is found to aggravate the ratelimiting strategy of DA synthesis pathway by increasing 
[3H]-DA synthesis [57, 58], tyrosine hydroxylase [59] and mRNA expression [60]. Similarly, in most rodent studies, demarcation with 3,4- Dihydroxyphenylacetic acid (DOPAC)/DA ratio also exhibited increased DA metabolism [61] despite other contradictory studies [59].

Contrary to the above findings, Acute THC, in rhesus monkeys, did not alter the protein levels in the DA receptor [62]. One study in the limbic forebrain of rats reported increased availability of DA type 1 receptor (D1R) [63] in contrast to other studies that showed decreased accessibility [64]. While, in the striatum, decreased or null significant changes in the density of DA type 2 receptor (D2R) was reported [64] with a decrease in D1R [63]. A study on human brain activity through functional magnetic resonance imaging (fMRI) and PET demonstrated that DAergic activity may alter due to the changes in glucose metabolism and cerebral blood blow. These serve as a substitute marker in areas with DAergic prognosis activity of the brain. Due to direct endocannabinoid effects, acute THC and high concentration of CB1R intensifies the activities in frontal and subcortical regions of the brain in humans $[65,66]$. Through a study in the striatum of a resting brain containing dense DAergic innervations, inconsistent effects were found pertaining to both elevated and reduced activity of acute THC [65]. However, DAergic signaling modulates certain cognitive ventures which may provide a vigorous substitution for DAergic transmission induced by THC. For instance, inhibition of motor response is accompanied by cortical DA release. Thereby, THC is found to attenuate its activity in the inferior frontal cortex (right) and anterior cingulated cortex (ACC) in humans previously exposed to cannabis [67]. A moderate DAergic activity was made evident in healthy humans previously exposed to cannabis; whereby THC induced striatal activation impairment [68]. In contrast, a study in occasional cannabis users, THC was found to induce a predominant impairment of brain responses in commentary reward trials [69]. Molecular imaging on the effect of acute THC on the DA system in humans with previous exposure to cannabis also revealed that THC stimulates DA release in the ventral striatum of the human brain [19].

\subsection{Cannabidiol (CBD) and Dopamine}

The first study on CBD induced anxiolytic and antipsychotic effects was demonstrated in the 1970s and 1980s and was later studied in humans executing promising results [70]. In addition to anxiety and psychotic effects, basic and clinical research on other therapeutic contributions of CBD was conducted. Moreover, synthetic analogs of CBD with efficacious potentiality have recently been developed recommended for patients with compromised health [71]. CBD was first isolated from cannabis extracts by Adams and his co-workers in the year 1940 [72]. In the early 1970s, several studies reported that $\mathrm{CBD}$ was incompetent to imitate the effects of cannabis, which led to hypothesize that; CBD would be a non-functioning cannabinoid. However, it was also speculated that $\mathrm{CBD}$, with other cannabinoids, could interfere with the THC effects $[73,74]$. These studies indicated the pharmacological activities of CBD exhibiting a broad spectrum of gesture [70].

Although, it is believed that CBD acts on DA receptors to induce its effects, there are no known reports that manifested its direct effect on D2 receptors of DA. Depending on this statement, a group of researchers recommended that CBD is the principal superficial exception in blocking or interfering DA at the D2 receptors of the brain DA system $[75,76]$. Previously, it was reported that the antipsychotic effects of CBD did not attribute to DAergic regions of the brain or any other brain receptor [77, 78]. Although it is a known fact that DA D2 receptors are the main target for antipsychotic drugs, it has been a prime factor of research on CBD to prove its antipsychotic property on $\mathrm{D} 2$ receptors of DA [76]. Experimental evidence helped to understand some of the effects of CBD that acts as an atypical antipsychotic drug [79-81]. $\triangle 9$-THC acts as a partial agonist at the G-protein-linked receptor at the rat cerebral cannabinoid, whereas CBD behaved as an antagonist at a range of micromolar concentrations [82].

Recent evidence suggested the antipsychotic effect of $\mathrm{CBD}$ as a promising potential antipsychotic treatment. In preclinical models of schizophrenia induced by psychometric drugs, $\mathrm{CBD}$ is found to reduce its detrimental effects by its neuropharmacological profile. As mentioned, CBD is found to be more effective than haloperidol and analogous to clozapine in attenuating ketamine-induced hyperlocomotion [80]. In experimental mice and rats, CBD has been reported to converse MK-801-induced sensorimotor gating deficits [83] and social withdrawal respectively [84]. In 2012, Leweke et al., documented in a recent clinical trial that, CBD possesses antipsychotic properties which reduce psychotic symptoms with significantly fewer side-effects [78]. However, the mechanisms underlying the antipsychotic effect of CBD is still unknown. Considerably, molecular evidence on schizophrenia explains disturbances in signaling pathways along with DA receptor function. These signaling pathways include the Wnt signal transduction pathway, Akt, GSK-3, and catenin. Most eminently, both typical and atypical medicaments can initiate these pathways [85-87].

Again, in a contemporary study, CBD is reported to attenuate AMPH-induced psychomotor sensitization and sensorimotor gating deficits [88]. They also intimated that CBD generates its effects through the modulation of mTOR/p70S6K signaling pathway phosphorylation in the NAc shells. Furthermore, CBD is documented to accustom the dysregulation of mesolimbic DA neuron activity states induced by AMPH. They also reported that CBD blocks 
modulation of mesolimbic DA and dysregulation of striatal activation patterns induced by acute THC. Corresponding to these findings, Englund et al. reported that pretreatment with CBD is potent against antipsychotic effects when assigned prior to the administration of THC [89]. However, Renard et al. [88] demonstrated a novel mechanism on the putative antipsychotic effects of CBD with the mesolimbic system. They justified their demonstration through animal models validated with DAergic sensitization. They reported CBD to attenuate AMPH-induced sensitization and activity of DAergic neurons within the ventral tegmental area (VTA) in the NAc shell. Additionally, they reported that CBD was dependent on the mTOR/p70S6K signaling pathway elevating its phosphorylation state and blocking these molecular effects in the NAc shell. It culminates that CBD directly acts on the mesolimbic pathway and DAergic functions which is a critical underlying variable in cannabis-induced psychometric complications [90, 91]. mTOR signaling is an analytical modulator of synaptic plasticity, neuronal morphology, and functional memory [92-94] and has been a growing interest of research over the past decades.

Preclinical studies accounted for a significant reduction in mTOR/p70S6K signaling in the PFC of patients experiencing depression [94], where, ketamine (noncompetitive NMDA receptor antagonist) imply rapid anti-depressant effect by modulating mTOR activation [95]. With the consistent findings, it can be disclosed that CBD as an antipsychotic medication can directly act upon DA D2 receptors of the striatum by increasing $\mathrm{mTOR} / \mathrm{p} 70 \mathrm{~S} 6 \mathrm{~K}$ signaling. As per the mechanism of CBD is concerned, the VTA and NAc equate a common association via DAergic and GABAergic afferents from the VTA and GABAergic efferents from subpopulations of NAc. The medium spiny neurons that evolved protrude back to the VTA [96-101]. The GABAergic projection is anticipated to arbitrate a "long-loop" inhibitory feedback to regulate VTA DA neurons [101-103].

\section{Cannabis in Medicinal Implementation}

In spite of the detrimental effects associated with cannabis use, the medical implications of the plant (Cannabis sativa) have gained much interest during the last 20 years [104]. The use of cannabis (marijuana) was accepted and validated in 1999 by the National Academies of Sciences, Engineering, and Medicine; and accordingly, managerial medical colleges recommended for its prescription to patients [105]. In 2017, an updated report called for a national research agenda, enhancement of research quality, up-gradation of collected data with surveillance endeavors, and strategies for an inscription of barriers in advancing the schedule of cannabis [106].
Over the last decade, in North America, there has been an increased interest in the use of medical cannabis. It is estimated that about 3.5 million people in the USA are purposely using medical cannabis legally, and is recorded for a total worth of USD \$ 6.7 billion are endowed in North America on legal marijuana in 2016 [107]. Health care of Canada approved the Canadian residents to purchase medical marijuana with prescriptions, which boosted its use from 30,537 in 2015 to near 100,000 in 2016 [108]. Medical cannabis assists the use of its components in the varied extent of medical conditions, remarkably in the field of pain management [109] and multiple sclerosis [110]. Numerous synthetic cannabinoids are useful in medicinal purposes and are already been produced viz. dronabinol and nabilone, which, in some ways, mimic the effects of THC. They are licensed by many countries including the US, Netherland, Germany, Austria for the treatment of weight loss in patients suffering from nausea, vomiting, and AIDS [16]. In comparison to other herbal remedies, the prepared content of non-medicinal CBD, as declared, is often inaccurate [111, $112]$, and these products periodically may exceed the legal limit of THC [112]. Moreover, when compared to the clinical trials [113], the amount of CBD in these products is found to be much lower $[114,115]$. There are some products composed of cannabis that were already available in the medicinal ground before its rescheduling in 2018. Sativex, derived from cannabis, is an oral spray that contains both THC and CBD in a 1:1 ratio. It is licensed in 29 countries including Canada and the UK and is used for the treatment of multiple sclerosis spasms. However, a meta-analysis suggested that its effectiveness may be inadequate and restricted [116]. Because of its weak and insufficient effectiveness, it is not recommended by the UK's National Institute for Health and Care Excellence (NICE) [117]. Additionally, a cannabis-derived oral solution named Epidiolex was licensed by the US Food and Drug Administration in 2018 for seizure medicaments in two rare and severe forms of childhood epilepsy-Lennox-Gaustat syndrome and Dravet syndrome [113].

Products of plant-derived cannabis are not well evaluated on the basis of traditional medicines, which led to an increase in uncertainty regarding its aptitude in human health [109]. While, it is well understood from these findings that there are many synthetic forms of cannabis that are easily accessible with prescriptions, but the various cannabis plant products obtained naturally differ in the concentrations of THC and CBD which makes the effects of exposure unpredictable [118].

\section{Discussion}

Cannabis is a known illicit drug that has been used for many decades. There are above 100 cannabinoids reported to be present, out of which THC is the causative incompetent 
cannabinoid that causes DA insult; whereas, $\mathrm{CBD}$ is a positively featured cannabinoid with antipsychotic effect. Behind these findings, the presence of other cannabinoids of cannabis is not yet isolated or introduced that could explain their possible effects on brain DA manifestations. This review highlights the evidence showing that THC exerts its detrimental effects on the DA system [19, 39, 40, 48]. The various research work and studies demonstrated that acute administration of THC elevated DA release and nerve activity which are region-specific and understanding their functional significance is the basic need. The available preclinical evidence suggested that the administration of chronic THC induces long-term complications on the DA system. There is a need for further studies on the use of cannabis in association with other drugs like nicotine and alcohol when administered together. There is also a need to understand the activities of other cannabinoids of Cannabis sativa, their role in the moderation of THC-induced DAergic alterations. Human PET studies have executed reports on blunted DA synthesis in cannabis users in contrast to non-users, the precise mechanisms underlying this process is not yet clear.

Besides the knowledge on the increasing use of medical cannabis in most countries, an understanding of the landscape of available evidence syntheses is needed to support results across reviews, including non-synthesized (studyby-study) data [107]. Many studies were unable to furnish a definitive statement regarding the positive repercussions of cannabis viz. pain management and multiple sclerosis [104]. Much research has been carried out on the hazardous effects of cannabis, providing relevance on THC menace, its jeopardy in DA synthesis and release, its effect in the DA receptors as well as NAc in regular cannabis users. Even so, the antipsychotic effect of CBD is not well evaluated in the DAergic region of the brain, which can elaborately explain its efficacy in DA release and its' relation with DA receptors.

\section{Conclusion and Future Perspectives}

Individuals may be influenced by an addiction arrest through various biological and sociological factors. This may induce vulnerability towards initial use in addition to the positive and negative reinforcement that follows. This vulnerability is further complicated with a comorbid psychiatric disorder and withdrawal of the same is followed by self-medication. The evidence highlighted in this study revealed the subversive effects of recreational cannabis rather than therapeutics. Evaluation and analysis of related findings is a measure to disseminate accurate information to the people so that individuals can resolve and make precocious choices on their use.

Precisely, acute exposure to both natural and synthetic cannabinoids engenders a complete array of transitory symptoms, cognitive deficits, and psychophysiological abnormalities. Eventually, cannabis exposure in adolescence yields a higher risk of psychosis, although dose-dependent. These relevant findings may contribute caution on cannabis-induced health criticism including global and specific domains of cognitive impairment which may be irreversible. Additional research and standard epidemiological studies are needed to further specify the extent of the adverse effects caused due to cannabis use.

Through these findings, it is evident that gestational exposure to THC assists dysregulation of DA synthesis which is proficient in potential public health implications. However, the reports from animal and human experiments on cannabis exposure exhibited conflict on the results. It is found that acute THC elevates DA release and neuronal activity, while long-term exposure to THC promotes a blunted DA system. Alternatively, CBD is found to ameliorate the psychotic symptoms induced by THC and has been reported to maintain DAergic sustenance.

However, behavioral consequences of THC and CBD along with the knowledge on their persistence in DAergic effects, whether long-term or short-term is not well established. Therefore, it can be suggested for supplementary research on the long-term and developmental DAergic effects of cannabis. Moreover, the pragmatic effects of CBD are not clearly justified on THC-induced psychosis and DA synthesis. Much research is still anticipated on these findings and effects of other cannabinoids on behavioral anomalies accompanying both physical alterations and brain DA systems.

Acknowledgements The authors sincerely acknowledge the funding (Sanction Order No. DST/Inspire Fellowship/2016/ IF160620) dated 01-22-2020 and support provided by the Department of Science and Technology, Govt. of India and University Grants Commission, New Delhi.

\section{Compliance with Ethical Standards}

Conflict of Interest The authors declare that they have no conflict of interest.

Open Access This article is licensed under a Creative Commons Attribution 4.0 International License, which permits use, sharing, adaptation, distribution and reproduction in any medium or format, as long as you give appropriate credit to the original author(s) and the source, provide a link to the Creative Commons licence, and indicate if changes were made. The images or other third party material in this article are included in the article's Creative Commons licence, unless indicated otherwise in a credit line to the material. If material is not included in the article's Creative Commons licence and your intended use is not permitted by statutory regulation or exceeds the permitted use, you will need to obtain permission directly from the copyright holder. To view a copy of this licence, visit http://creativecommons.org/licenses/by/4.0/. 


\section{References}

1. G. Thomas, R.A. Kloner, S. Rezkalla, Adverse cardiovascular, cerebrovascular, and peripheral vascular effects of marijuana inhalation: what cardiologists need to know. Am. J. Cardiol. 133, 187-190 (2014)

2. V. Wolff, E. Jouanjus, Strokes are possible complications of cannabinoids use. Epilepsy Behav. 70, 356-363 (2017)

3. United Nations Office on Drugs and Crime (UNODC), Cannabis and hallucinogens, World Drug Rep. 2019. p. 1-71.

4. T.C. Ford, A.C. Hayley, L.A. Downey, A.C. Parrott, Cannabis: an overview of its adverse acute and chronic effects and its implications. Curr. Drug Abuse Rev. 10, 6-8 (2017)

5. L. Karila, P. Roux, B. Rolland, A. Benyamina, M. Reynaud, H.J. Aubin, C. Lancon, Acute and long term effects of cannabis use: a review. Curr. Pharm. Des. 20, 4112-4118 (2014)

6. C.M. Milroy, J.L. Parai, The histopathology of drugs of abuse. Histopathology 59, 579-593 (2011)

7. B.R. Thanvi, S.D. Treadwell, Cannabis and Stroke: is there a link? Postgrad. Med. J. 85, 80-83 (2009)

8. B. Chadwick, M.L. Miller, Y.L. Hurd, Cannabis use during adolescent development: susceptibility to psychiatric illness. Front. Psychiatry. 4, 129 (2013)

9. G. Battistella, E. Fornari, J.M. Annoni, M. Chtioui, K. Dao, M. Fabritius, B. Favrat, J.F. Mall, P. Maeder, C. Giroud, Long term effects of cannabis on brain structure. Neuropsychopharmacology. 39, 2041-2048 (2014)

10. S.J. Broyd, H.H. Van Hell, C. Beale, M. Yueel, N. Solouiji, Acute and chronic effects of cannabinoids on human cognition- a systematic review. Biol. Psychiatry. 79, 57-567 (2016)

11. H. Szutorisz, Y.L. Hurd, High times for cannabis: epigenetic imprint and its legacy on brain and behavior. Neurosci. Biobehav. Rev. 85, 93-101 (2018)

12. S. Chandra, M.M. Radwan, C.G. Majumdar, J.C. Church, T.P. Freeman, M.A. ElSohly, New trends in cannabis potency in USA and Europe during the last decade (2008-2017). Eur. Arch. Psychiatry Clin. Neurosci. 269, 5-15 (2019)

13. Z. Atakan, Cannabis, a complex plant: different compounds and different effects on individuals. Ther. Adv. Psychopharmacol. 2, 241-254 (2012)

14. T.A. Iseger, M.G. Bossong, A systematic review of the antipsychotic properties of cannabidiol in humans. Schizophrenia Res. 162, 1-3 (2015)

15. A. Batalla, J.A. Crippa, G.F. Busatto, F.S. Guimarae, A.W. Zuardi, O. Valverde, Z. Atakan, P.K. McGuire, S. Bhattacharyya, R. Martin-Santos, Neuroimaging studies of acute effects of THC and CBD in humans and animals: a systematic review. Curr. Pharm. Des. 20, 2168-2185 (2014)

16. T.P. Freeman, C. Hindocha, S.F. Green, M.A.P. Bloomfield, Medical use of cannabis based products and cannabinoids. BMJ 365, 1-7 (2019)

17. J.M.N.C. Ton, G.A. Gerhardt, M. Friedemann, A.M. Etgen, G.M. Rose, N.S. Sharpless, E.L. Gardner, The effects of delta 9 tetrahydrocannabinol on potassium-evoked release of dopamine in the rat caudate nucleus: an in vivo electrochemical and in vivo microdialysis study. Brain Res. 451, 59-68 (1988)

18. P.R.A. Stokes, A. Egerton, B. Watson, A. Reid, G. Breen, A. Lingford-Hughes, D.J. Nutt, M.A. Mehta, Significant decreases in frontal and temporal [11C]-raclopride binding after THC challenge. NeuroImage. 52, 1521-1527 (2010)

19. M.G. Bossong, M.A. Mehta, B.N.M. VanBerckel, O.D. Howes, R.S. Kahn, P.R.A. Stokes, Further human evidence for striatal dopamine release induced by administration of 89-tetrahydrocannabinol (THC): selectivity to limbic striatum. Psychopharmacology 232, 2723-2729 (2015)

20. M.A.P. Bloomfield, A.H. Ashok, N.D. Volkow, O.D. Howes, The effects of $\Delta 9$-tetrahydrocannabinol on the dopamine system. Nature 539, 369-377 (2016)

21. G.F. Koob, N.D. Volkow, Neurobiology of addiction: a neurocircuitry analysis. Lancet Psychiatry. 3, 760-773 (2016)

22. P. Trifilieff, D. Martinez, Imaging addiction: D2 receptors and dopamine signaling in the striatum as biomarkers for impulsivity. Neuropharmacol. 76, 498-509 (2014)

23. N.D. Volkow, J.S. Fowler, G.J. Wang, R. Baler, F. Telang, Imaging dopamine's role in drug abuse and addiction. Neuropharmacol. 56, 3-8 (2009)

24. M.A. Bloomfield, C.J. Morgan, A. Egerton, S. Kapur, H.V. Curran, O.D. Howes, Dopaminergic function in cannabis users and its relationship to cannabis-induced psychotic symptoms. Biol. Psychiatry. 75, 470-478 (2014)

25. R. Mizrahi, M. Kenk, I. Suridjan, I. Boileau, T.P. George, K. McKenzie, A.A. Wilson, S. Houle, P. Rusjan, Stress-induced dopamine response in subjects at clinical high risk for schizophrenia with and without concurrent cannabis use. Neuropsychopharmacol 39, 1479-1489 (2014)

26. D.M. Dougherty, C.W. Mathias, M.A. Dawes, R.M. Furr, N.E. Charles, A. Liguori, E.E. Shannon, A. Acheson, Impulsivity, attention, memory, and decision-making among adolescent marijuana users. Psychopharmacol. 226, 307-319 (2013)

27. S.M. Landau, R. Lal, J.P. O’Neil, S. Baker, W.J. Jagust, Striatal dopamine and working memory. Cereb. Cortex. 19, 445-454 (2009)

28. L. Wilkinson, Y.F. Tai, C.S. Lin, D.A. Lagnado, D.J. Brooks, P. Piccini, M. Jahanshahi, Probabilistic classification learning with corrective feedback is associated with in vivo striatal dopamine release in the ventral striatum, while learning without feedback is not. Hum. Brain Mapp. 35, 5106-5115 (2014)

29. E. van de Giessen, J.J. Weinstein, C.M. Cassidy, M. Haney, Z. Dong, R. Ghazzaoui, N. Ojeil, L.S. Kegeles, X. Xu, N.P. Vadhan, N.D. Volkow, M. Slifstein, A. Abi-Dargham, Deficits in striatal dopamine release in cannabis dependence. Mol. Psychiatry. 22, 68-75 (2017)

30. C. de la Fuente-Sandoval, P. Leon-Ortiz, R. Favila, S. Stephano, D. Mamo, J. Ramirez-Bermudez, A.G. Guerrero, Higher levels of glutamate in the associative-striatum of subjects with prodromal symptoms of schizophrenia and patients with first-episode psychosis. Neuropsychopharmacol 36, 1781-1791 (2011)

31. N.V. Kraguljac, D.M. White, M.A. Reid, A.C. Lahti, Increased hippocampal glutamate and volumetric deficits in unmedicated patients with schizophrenia. JAMA Psychiatry. 70, 1294-1302 (2013)

32. D.M. Semple, A.M. McIntosh, S.M. Lawrie, Cannabis as a risk factor for psychosis: systematic review. J. Psychopharmacol. 19, 187-194 (2005)

33. J. Ceccarini, M. De Hert, R. Van Winkel, J. Peuskens, G. Bormans, L. Kranaster, F. Enning, D. Koethe, F.M. Leweke, K.V. Laere, Increased ventral striatal CB1 receptor binding is related to negative symptoms in drug-free patients with schizophrenia. NeuroImage. 79, 304-312 (2013)

34. A.B. Martin, E. Fernandez-Espejo, B. Ferrer, M.A. Gorriti, A. Bilbao, M. Navarro, F.R. de Fonseca, R.R. Moratalla, Expression and function of CB1 receptor in the rat striatum: localization and effects on D1 and D2 dopamine receptor-mediated motor behaviors. Neuropsychopharmacol 33, 1667-1679 (2008)

35. V. Van Waes, J.A. Beverley, H. Siman, K.Y. Tseng, H. Steiner, CB1 Cannabinoid receptor expression in the striatum: association with corticostriatal circuits and developmental regulation. Front. Pharmacol. 3, 21 (2012) 
36. M. Herkenham, A.B. Lynn, M.D. Little, M.R. Johnson, L.S. Melvin, B.R. de Costa, K.C. Rice, Cannabinoid receptor localization in brain. Proceed. Nat. Acad. Sci. U. S. A. 87, 1932-1936 (1990)

37. C.C. Felder, J.S. Veluz, H.L. Williams, E.M. Briley, L.A. Matsuda, Cannabinoid agonists stimulate both receptor- and nonreceptor-mediated signal transduction pathways in cells transfected with and expressing cannabinoid receptor clones. Mol. Pharmacol. 42, 838-845 (1992)

38. C.R. Lupica, A.C. Riegel, A.F. Hoffman, Marijuana and cannabinoid regulation of brain reward circuits. Br. J. Pharmacol. 143, 227-234 (2004)

39. M.G. Bossong, B.N. van Berckel, R. Boellaard, L. Zuurman, R.C. Schuit, A.D. Windhorst, J.M.A. van Gerven, N.F. Ramsey, A.A. Lammertsma, R.S. Kahn, Delta 9tetrahydrocannabinol induces dopamine release in the human striatum. Neuropsychopharmacol 34, 759-766 (2009)

40. L.N. Voruganti, P. Slomka, P. Zabel, A. Mattar, A.G. Awad, Cannabis induced dopamine release: an invivo SPECT study. Psychiatry Res. 107, 173-177 (2001)

41. P.R. Stokes, M.A. Mehta, H.V. Curran, G. Breen, P.M. Grasby, Can recreational doses of THC produce significant dopamine release in the human striatum? NeuroImage. 48, 186-190 (2009)

42. E. Barkus, P.D. Morrison, D. Vuletic, J.C. Dickson, P.J. Ell, L.S. Pilowsky, R. Brenneisen, D.W. Holt, J. Powell, S. Kapur, R.M. Murray, Does intravenous Delta9-tetrahydrocannabinol increase dopamine release? A SPET study. J. Psychopharmacol. 25, 1462-1468 (2011)

43. M. Vallee, Pregnenolone can protect the brain from cannabis intoxication. Science 343, 94-98 (2014)

44. M.A. Huestis, D.A. Gorelick, S.J. Heishman, K.L. Peston, R.A. Nelson, E.T. Moolchan, R.A. Frank, Blockade of effects of smoked marijuana by the CB1-selective cannabinoid receptor antagonist SR141716. Arch. Gen. Psychiatry 58, 322-328 (2001)

45. M.R. Elphick, M. Egertova, The neurobiology and evolution of cannabinoid signalling. Philos Trans R Soc Lond B Biol Sci. 356, 381-408 (2001)

46. R.G. Pertwee, The diverse $\mathrm{CB} 1$ and $\mathrm{CB} 2$ receptor pharmacology of three plant cannabinoids: delta9tetrahydrocannabinol, cannabidiol and delta9-tetrahydrocannabivarin. Br. J. Pharmacol. 153, 199-215 (2008)

47. M. Kathmann, K. Flau, A. Redmer, C. Trankle, E. Schlicker, Cannabidiol is an allosteric modulator at mu- and delta-opioid receptors. Naunyn-Schmiedeberg's Archiv. Pharmacol. 372, 354-361 (2006)

48. E.H. Chartoff, H.S. Connery, It's MORe exciting than mu: crosstalk between mu opioid receptors and glutamatergic transmission in the mesolimbic dopamine system. Front Pharmacol. 5, 116 (2014)

49. J.C. Garriott, L.J. King, R.B. Forney, F.W. Hughes, Effects of some tetrahydrocannabinols on hexobarbital sleeping time and amphetamine induced hyperactivity in mice. Life Sci. 6, 21192128 (1967)

50. J. Howes, P. Osgood, The effect of delta9-tetrahydrocannabinol on the uptake and release of 14Cdopamine from crude striatal synaptosoma; preparations. Neuropharmacol. 13, 1109-1114 (1974)

51. J. Fernandez-Ruiz, M. Hernandez, J.A. Ramos, Cannabinoiddopamine interaction in the pathophysiology and treatment of CNS disorders. CNS Neurosci. Ther. 16, 72-91 (2010)

52. M. Herkenham, A.B. Lynn, B.R. Decosta, E.K. Richfield, Neuronal localization of cannabinoid receptors in the basal ganglia of the rat. Brain Res. 547, 267-274 (1991)

53. M.A. De Luca, V. Valentini, Z. Bimpisidis, F. Cacciapaglia, P. Caboni, G. Di Chiara, Endocannabinoid 2-arachidonoylglycerol self-administration by SpragueDawley rats and stimulation of in vivo dopamine transmission in the nucleus accumbens shell. Front. Psychiatry. 5, 140 (2014)

54. A.S. Bloom, W.L. Dewey, A comparison of some pharmacological actions of morphine and delta 9 tetrahydrocannabinol in the mouse. Psychopharmacol. 57, 243-248 (1978)

55. M. Hershkowitz, H. Szechtman, Pretreatment with delta 1-tetrahydrocannabinol and psychoactive drugs: effects on uptake of biogenic amines and on behavior. Euro. J. Pharmacol. 59, 267-276 (1979)

56. M.K. Poddar, W.L. Dewey, Effects of cannabinoids on catecholamine uptake and release in hypothalamic and striatal synaptosomes. J. Pharmacol. Exp. Ther. 214, 63-67 (1980)

57. L. Maitre, M. Staehelin, H.J. Bein, Effect of an extract of cannabis and of some cannabinols on catecholamine metabolism in rat brain and heart. Agents Actions 1, 136-143 (1970)

58. A.S. Bloom, K.M. Johnson, W.L. Dewey, The effects of cannabinoids on body temperature and brain catecholamine synthesis. Res. Commun. Chem. Pathol. Pharmacol. 20, 51-57 (1978)

59. J. Romero, R. Demiguel, E. Garciapalomero, J.J. Fernandezruiz, J.A. Ramos, Time-course of the effects of anandamide, the putative endogenous cannabinoid receptor-ligand, on extrapyramidal function. Brain Res. 694, 223-232 (1995)

60. B. Bosier, G.G. Muccioli, B. Mertens, S. Sarre, Y. Michotte, D.M. Lambert, E. Hermans, Differential modulations of striatal tyrosine hydroxylase and dopamine metabolism by cannabinoid agonists as evidence for functional selectivity in vivo. Neuropharmacol. 62, 2328-2336 (2012)

61. M. Navarro, J.J. Fernandez-Ruiz, R. de Miguel, M.L. Hernandez, M. Cebeira, J.A. Ramos, An acute dose of delta9-tetrahydrocannabinol affects behavioral and neurochemical indices of mesolimbic dopaminergic activity. Behav. Brain Res. 57, 37-46 (1993)

62. S.F. Ali, G.D. Newport, A.C. Scallet, M.G. Paule, J.R. Bailey, W. Slikker Jr., Chronic marijuana smoke exposure in the rhesus monkey. IV: neurochemical effects and comparison to acute and chronic exposure to delta-9-tetrahydrocannabinol (THC) in rats. Pharmacol. Biochem. Behav. 40, 677-682 (1991)

63. M. Navarro, J.J. Fernandez-Ruiz, R. de Miguel, M.L. Hernandez, M. Cebeira, J.A. Ramos, Motor disturbances induced by an acute dose of DELTA9-tetrahydrocannabinol: possible involvement of nigrostriatal dopaminergic alterations. Pharmacol. Biochem. Behav. 45, 291-298 (1993)

64. F.R. Defonseca, J.J. Fernandez-Ruiz, L.L. Murphy, M. Cebeira, R.W. Steger, A. Bartke, J.A. Ramos, acute effects of delta-9-tetrahydrocannabinol on dopaminergic activity in several rat-brain areas. Pharmacol. Biochem. Behav. 42, 269-275 (1992)

65. N.D. Volkow, H. Gillespie, N. Mullani, L. Tancredi, C. Grant, A. Valentine, L. Hollister, Brain glucose metabolism in chronic marijuana users at baseline and during marijuana intoxication. Psychiatry Res. 67, 29-38 (1996)

66. R.G. Pertwee, R.A. Ross, Cannabinoid receptors and their ligands. Prostaglandins Leukot. Essent. Fatty Acids. 66, 101-121 (2002)

67. S.J. Borgwardt, P. Allen, S. Bhattacharyya, P. Fusar-Poli, J.A. Crippa, M.L. Seal, V. Fraccaro, Z. Atakan, R. Martin-Santos, C. O'Carroll, K. Rubia, P.K. McGuire, Neural basis of Delta9-tetrahydrocannabinol and cannabidiol: effects during response inhibition. Biol. psychiatry. 64, 966-973 (2008)

68. S. Bhattacharyya, P.D. Morrison, P. Fusar-Poli, R. Martin-Santos, S. Borgwardt, T. Winton-Brown, C. Nosarti, C.M. O'Carroll, M. Seal, P. Allen, M.A. Mehta, J.M. Stone, N. Tunstall, V. Giampietro, S. Kapur, R.M. Murray, A.W. Zuardi, J.A. Crippa, Z. Atakan, P.K. McGuire, Opposite effects of delta-9-tetrahydrocannabinol and cannabidiol on human brain function and psychopathology. Neuropsychopharmacol 35, 764-774 (2010) 
69. H.H. van Hell, G. Jager, M.G. Bossong, A. Brouwer, J.M. Jansma, L. Zuurman, J. van Gerven, R.S. Kahn, N.F. Ramsey, Involvement of the endocannabinoid system in reward processing in the human brain. Psychopharmacol. 219, 981-990 (2012)

70. A.W. Zuardi, Cannabidiol: from an inactive cannabinoid to a drug with wide spectrum of action. Rev. Bras. Psiquiatr. 30, 271-280 (2008)

71. R. Mechoulam, A.W. Zuardi, F.P. Kapczinski, J.E.C. Hallak, F.S. Guimaraes, J.A.S. Crippa, A. Breuer, Fluorinated CBD Compounds, Compositions and Uses Thereof. Pub. No.: WO/2014/ 108899. International Application No.: PCT/IL2014/050023. (2014)

72. R. Adams, M. Hunt, J.H. Clark, Structure of cannabidiol, a product isolated from the marihuana extract of Minnesota wild hemp. J. Am. Chem. Soc. 62, 196-200 (1940)

73. E.A. Carlini, M. Santos, U. Claussen, D. Bieniek, F. Korte, Structure activity relationship of four tetrahydrocannabinols and the pharmacological activity of five semi-purified extracts of Cannabis sativa. Psychopharmacol. 18, 82-93 (1970)

74. I.G. Karniol, E.A. Carlini, The content of (-) 9 -trans-tetrahydrocannabinol (9-thc) does not explain all biological activity of some Brazilian marihuana samples. J. Pharm. Pharmacol. 24, 833-834 (1972)

75. P. Seeman, M. Chau-Wong, J. Tedesco, K. Wong, Brain receptors for antipsychotic drugs and dopamine: direct binding assays. Proc. Natl. Acad. Sci. U. S. A. 72, 4376-4380 (1975)

76. P. Seeman, All roads to schizophrenia lead to dopamine supersensitivity and elevated dopamine D2High receptors. CNS Neurosci. Ther. 17, 118-132 (2011)

77. L. Davenport, Cannabis compound may augment antipsychotic meds. Available at www.medscape.com/viewarticle/862312 (accessed on 9 May 2016)

78. F.M. Leweke, D. Piomelli, F. Pahlisch, D. Muhl, C.W. Gerth, C. Hoyer, J. Klosterkotter, M. Hellmich, D. Koethe, Cannabidiol enhances anandamide signaling and alleviates psychotic symptoms of schizophrenia. Transl. Psychiatry. 2, e94 (2012)

79. A.W. Zuardi, J.A. Rodrigues, J.M. Cunha, Effects of cannabidiol in animal models predictive of antipsychotic activity. Psychopharmacol. (Berl) 104, 260-264 (1991)

80. F.A. Moreira, F.S. Guimarães, Cannabidiol inhibits the hyperlocomotion induced by psychotomimetic drugs in mice. Eur. J. Pharmacol. 512, 199-205 (2005)

81. V.M. Guimarães, A.W. Zuardi, E.A. Del Bel, F.S. Guimarães, Cannabidiol increases Fos expression in the nucleus accumbens but not in the dorsal striatum. Life Sci. 75, 633-638 (2004)

82. F. Petitet, B. Jeantaud, M. Reibaud, A. Imperato, M.C. Dubroeucq, Complex pharmacology of natural cannabinoids: evidence for partial agonist activity of delta9tetrahydrocannabinol and antagonist activity of cannabidiol on rat brain cannabinoid receptors. Life Sci. 63, PL1-PL6 (1998)

83. L.E. Long, D.T. Malone, D.A. Taylor, Cannabidiol reverses MK-801 induced disruption of prepulse inhibition in mice. Neuropsychopharmacol. 31, 795-803 (2006)

84. A. Gururajan, D.A. Taylor, D.T. Malone, Effect of cannabidiol in a MK801-rodent model of aspects of Schizophrenia. Behav. Brain Res. 222, 299-308 (2011)

85. H. Alimohamad, L. Sutton, J. Mouyal, N. Rajakumar, W.J. Rushlow, The effects of antipsychotics on B-catenin, glycogen synthase kinase- 3 and disheveled in the ventral midbrain of rats. J. Neurochem. 95, 513-525 (2005)

86. L.P. Sutton, D. Honardoust, J. Mouyal, N. Rajakumar, W.J. Rushlow, Activation of the canonical Wnt pathway by the antipsychotics haloperidol and clozapine involves dishevelled-3. J. Neurochem. 102, 153-169 (2007)
87. Z. Freyberg, S.J. Ferrando, J.A. Javitch, Roles of the Akt/GSK-3 and Wnt signaling pathways in schizophrenia and antipsychotic drug action. Am. J. Psychiatry. 167, 388-396 (2010)

88. J. Renard, M. Loureiro, L.G. Rosen, J. Zunder, C. de Oliveira, S. Schmid, W.J. Rushlow, S.R. Laviolette, Cannabidiol counteracts amphetamine-induced neuronal and behavioral sensitization of the mesolimbic dopamine pathway through a novel mTOR/ p70S6 kinase signaling pathway. J. Neurosci. 36, 5160-5169 (2016)

89. A. Englund, P.D. Morrison, J. Nottage, D. Hague, F. Kane, S. Bonaccorso, J.M. Stone, A. Reichenberg, R. Brenneisen, D. Holt, A. Feilding, L. Walker, R.M. Murray, S. Kapur, Cannabidiol inhibits THC-elicited paranoid symptoms and hippocampaldependent memory impairment. J. Psychopharmacol. 27, 19-27 (2013)

90. M. Di Forti, J.M. Lappin, R.M. Murray, Risk factors for schizophrenia - all roads lead to dopamine. Eur. Neuropsychopharmacol. 17(Suppl 2), S101-S107 (2007)

91. S. Luzi, P.D. Morrison, J. Powell, M. di Forti, R.M. Murray, What is the mechanism whereby cannabis use increases risk of psychosis? Neurotox. Res. 14, 105-112 (2008)

92. M.D. Antion, M. Merhav, C.A. Hoeffer, G. Reis, S.C. Kozma, G. Thomas, E.M. Schuman, K. Rosenblum, E. Klann, Removal of S6K1 and S6K2 leads to divergent alterations in learning, memory, and synaptic plasticity. Learn. Mem. 15, 29-38 (2008)

93. C.A. Hoeffer, E. Klann, mTOR signaling: at the crossroads of plasticity, memory and disease. Trends Neurosci. 33, 67-75 (2010)

94. C.S. Jernigan, D.B. Goswami, M.C. Austin, A.H. Iyo, A. Chandran, C.A. Stockmeier, B. Karolewicz, The mTOR signaling pathway in the prefrontal cortex is compromised in major depressive disorder. Prog. Neuropsycho. Pharmacol. Biol. Psychiatry. 35, 1774-1779 (2011)

95. N. Li, B. Lee, R.J. Liu, M. Banasr, J.M. Dwyer, M. Iwata, X.Y. Li, G. Aghajanian, R.S. Duman, mTOR-dependent synapse formation underlies the rapid antidepressant effects of NMDA antagonists. Science 329, 959-964 (2010)

96. P.W. Kalivas, P. Duffy, Time course of extracellular dopamine and behavioral sensitization to cocaine: II dopamine perikarya. J. Neurosci. 13, 276-284 (1993)

97. X.Y. Lu, M.B. Ghasemzadeh, P.W. Kalivas, Expression of D1 receptor, D2receptor, substance Pandenkephalin messenger RNAs in the neurons projecting from the nucleus accumbens. Neuroscience 82, 767-780 (1998)

98. I. Usuda, K. Tanaka, T. Chiba, Efferent projections of the nucleus accumbens in the rat with special reference to subdivision of the nucleus: biotinylated dextranamine study. Brain Res. 797, 73-93 (1998)

99. D.B. Carr, S.R. Sesack, Projections from the rat prefrontal cortex to the ventral tegmental area: target specificity in the synaptic associations with mesoaccumbens and mesocortical neurons. J. Neurosci. 20, 3864-3873 (2000)

100. A. Tripathi, L. Prensa, C. Cebrián, E. Mengual, Axonal branching patterns of nucleus accumbens neurons in the rat. J. Comp. Neurol. 18, 4649-4673 (2010)

101. Y. Xia, J.R. Driscoll, L. Wilbrecht, E.B. Margolis, H.L. Fields, G.O. Hjelmstad, Nucleus accumbens medium spiny neurons target nondopaminergic neurons in the ventral tegmental area. J. Neurosci. 31, 7811-7816 (2011)

102. L.C. Einhorn, P.A. Johansen, F.J. White, Electrophysiological effects of cocaine in the mesoaccumbens dopamine system: studies in the ventral tegmental area. J. Neurosci. 8, 100-112 (1988)

103. S. Rahman, W.J. McBride, Feedback control of mesolimbic somatodendritic dopamine release in rat brain. J. Neurochem. 74, 684-692 (2000) 
104. M. Pratt, A. Stevens, M. Thuku, C. Butler, B. Skidmore, L.S. Wieland, M. Clemons, S. Kanji, B. Hutton, Benefits and harms of medical cannabis: a scoping review of systematic reviews. Systemat. Rev. 8, 320 (2019)

105. S.J. Watson, J.A. Benson, J.E. Joy, Marijuana and medicine: assessing the science base: a summary of the 1999, Institute of Medicine report. Arch. Gen. Psychiatry. 57, 547-552 (2000)

106. National Academies of Sciences, Engineering, and Medicine, Health and Medicine Division, Board on Population Health and Public Health Practice \& Committee on the Health Effects of Marijuana: An Evidence Review and Research Agenda. The health effects of cannabis and cannabinoids: the current state of evidence and recommendations for research. US: National Academies Press; (2017)

107. Number of Legal Medical Marijuana Patients - Medical Marijuana - ProCon. org. https://medicalmarijuana.procon.org/view. resource.php? resourceID $=005889$

108. J. Miller, Number of Canadians buying legal medical marijuana triples in past year, Ottawa Citizen. https://ottawacitizen.com/ news/local-news/number-of-canadians-buying-legal-medicalmar ijuana-triples-in-just-one-year (2016)

109. W. Häuser, F. Petzke, M.A. Fitzcharles, Efficacy, tolerability and safety of cannabis-based medicines for chronic pain management-an overview of systematic reviews. Eur. J. Pain Lond. Engl. 22, 455-470 (2018)

110. S. Herzog, M. Shanahan, P. Grimison, A. Tran, N. Wong, N. Lintzeris, J. Simes, M. Stockler, R.L. Morton, Systematic review of the costs and benefits of prescribed cannabis-based medicines for the management of chronic illness: lessons from multiple sclerosis. Pharmacoeconom. 36, 67-78 (2017)

111. R. Pavlovic, G. Nenna, L. Calvi, S. Panseri, G. Borgonovo, L. Giupponi, G. Cannazza, A. Giorgi, Quality traits of "cannabidiol oils": cannabinoids content, terpene fingerprint and oxidation stability of European commercially available preparations. Molecules 23, 1230 (2018)

112. A. Hazekamp, The trouble with CBD oil. Med. Cannabis Cannabinoids 1, 65-72 (2018)

113. O. Devinsky, A.D. Patel, E.A. Thiele, M.H. Wong, R. Appleton, C.L. Harden, S. Greenwood, G. Morrison, K. Sommerville, GWPCARE1 Part A study group. Randomized, dose-ranging safety trial of cannabidiol in Dravet syndrome. Neurol. 90, e1204-e1211 (2018)

114. S. Shannon, J. Opila-Lehman, Effectiveness of cannabidiol oil for pediatric anxiety and insomnia as part of posttraumatic stress disorder: a case report. Perm. J. Fall. 20, 16-005 (2016)

115. S. Shannon, J. Opila-Lehman, Cannabidiol oil for decreasing addictive use of marijuana: a case report. Integr. Med. (Encinitas) 14, 31-35 (2015)

116. P.F. Whiting, R.F. Wolff, S. Deshpande, M.D. Nisio, S. Duffy, A.V. Harnandez, J.C. Keurentjes, S. Lang, K. Misso, S. Ryder, S. Schmidlkofer, M. Westwood, J. Kleijnen, Cannabinoids for medical use: a systematic review and meta-analysis. JAMA 313, 2456-2473 (2015)

117. National Institute for Health and Care Excellence. Do Not Do Recommendations: Sativex to treat spasticity in people with MS because it is not a cost effective treatment. 2014. https://www. nice.org.uk/donotdo/do-not-offer-sativex-to-treat-spasticity-inpeople-withms-because-it-is-not-a-cost-effective-treatment.

118. M. Colizzi, S. Bhattacharyya, Does cannabis composition matter? Differential effects of delta-9-tetrahydrocannabinol and cannabidiol on human cognition. Curr. Addict. Rep. 4, 62-74 (2017) 